XIV Simpósio Nacional de Sistemas Prediais

Gestão, Eficiência e Sustentabilidade

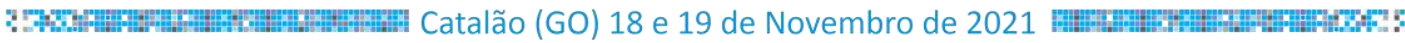

\title{
DECAIMENTO DE TEMPERATURA EM TUBULAÇÕES DE PPR PN 25 DE DIFERENTES DIÂMETROS PARA CONDUÇÃO DE ÁGUA QUENTE
}

\section{Temperature decay in PPR PN 25 pipes of different diameters for conducting hot water}

\author{
UEHARA, Takashi ${ }^{1}$; NASCIMENTO, César Henrique Britto ${ }^{2}$; FERREIRA, Armando Traini ${ }^{3}$ \\ Recebido em 15 de julho de 2021, aprovado em 06 de setembro de 2021, publicado em 18 de novembro de 2021
}

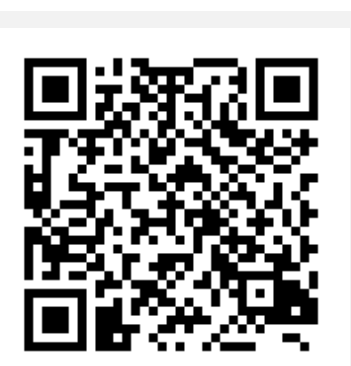

Palavras-chave:

Decaimento de

Temperatura,

Polipropileno

Copolímero Random,

PPR PN 25.

Keywords:

Temperature Decay,

Random Copolymer

Polypropylene,

PPR PN 25.

\begin{abstract}
RESUMO: O Polipropileno Copolímero Random (PPR) é um material muito usado em instalações prediais e industriais de água fria e quente. No transporte de água quente, a água perde temperatura entre o início e o fim de um trecho de tubulação analisado, o que ocasiona perda de energia em forma de calor para o ambiente. Este trabalho analisa o decaimento de temperatura em tubulações de PPR PN 25 nos diversos diâmetros existentes, com métodos iterativos e simulações computacionais. Os resultados obtidos no Excel e no Ansys Fluent apresentaram diferenças menores que $0,2 \%$, sendo que as maiores diferenças $(0,154 \%)$ ocorreram na análise com regime turbulento no Ansys Fluent. Este artigo apresenta resultados preliminares da pesquisa que necessitará de mais estudos para alcançar resultados mais precisos.
\end{abstract}

\begin{abstract}
Polypropylene Copolymer Random (PPR) is a material widely used in building and industrial installations of cold and hot water. In the transport of hot water, the water loses temperature between the beginning and the end of an analyzed pipe section, which causes loss of energy in the form of heat to the environment. In this paper it was analyzed the temperature decay in PPR PN 25 pipes in the various existing diameters, using iterative methods and computational simulations. The results obtained in Excel and Ansys Fluent showed differences lower than $0.2 \%$, and the largest differences $(0.154 \%)$ occurred in the turbulent regime analysis in Ansys Fluent. This article presents preliminary results of the research that will need further studies to achieve more accurate results.
\end{abstract}

CONTATO DOS AUTORES:

1 UEHARA, Takashi. Estudante de Engenharia Civil. Instituto Federal de Educação, Ciência e Tecnologia de São Paulo - Campus São Paulo. takashi.u@aluno.ifsp.edu.br.

${ }^{2}$ NASCIMENTO, César Henrique Britto. Estudante de Engenharia Civil. Instituto Federal de Educação, Ciência e Tecnologia de São Paulo - Campus São Paulo. cesarbritto13@gmail.com.

${ }^{3}$ FERREIRA, Armando Traini. Engenheiro Civil, Doutor, Professor do Instituto Federal de Educação, Ciência e Tecnologia de São Paulo - Campus São Paulo. traini@ifsp.edu.br. 


\section{INTRODUÇÃO}

Conforme Danieletto (2007), o polipropileno é obtido pela "polimerização do propileno, em reator na presença de solventes, catalizadores, hidrogênio e comonômeros quando for o caso. O catalizador entra no processo para desencadear a reação e propiciar a estereoespecificidade, ou seja, fazer com que as unidades básicas se interliguem na conformação desejada (daí o termo catalizador estereosespecífico), enquanto o hidrogênio $\left(\mathrm{H}_{2}\right)$ entra como elemento finalizador ou limitador de comprimento de macromoléculas (...). Os polipropilenos copolímeros randômicos possuem os grupos $\mathrm{C}_{3} \mathrm{H}_{6}$ e os grupos $\mathrm{C}_{2} \mathrm{H}_{4}$ de forma aleatória, não caracterizando blocos".

Ao conduzir água quente, há um decaimento de temperatura ao longo de uma tubulação, ocasionando perda de energia em forma de calor para o ambiente. Por esse motivo, é de grande importância o uso de sistemas que minimizem o decaimento de temperatura da água para evitar a necessidade de um aumento no consumo de energia a fim de atender a temperatura requerida para utilização.

O Polipropileno Copolímero Random (PPR) é uma resina poliolefinica que possui baixa condutividade térmica sendo, portanto, um isolante térmico. Diante disso, este material é muito usado para fabricação de tubos condutores de água quente, visto que a baixa condutividade térmica permite um menor decaimento de temperatura do fluido transportado.

Este trabalho justifica-se pela necessidade de maiores informações sobre o desempenho do tubo de PPR PN 25 na condução de água quente, pois não consta, nos catálogos dos fabricantes dessa tubulação, o gráfico do decaimento de temperatura em função do diâmetro, sendo essa uma informação importante para os projetistas hidráulicos.

\section{OBJETIVO}

O objetivo deste trabalho é determinar o decaimento de temperatura no transporte de água quente nos tubos de PPR nas diferentes configurações de diâmetro disponíveis, considerando a classe de pressão PN $25\left(25 \mathrm{kgf} / \mathrm{cm}^{2}\right)$.

\section{FUNDAMENTAÇÃO}

Segundo Borgnakke e Sonntag (2018), o calor é definido como a transferência de energia de um sistema, em uma dada temperatura, para outro, com temperatura inferior. Há três modos de transferência de calor em um meio ou entre meios: condução, convecção e radiação. A condução se dá através de meios estacionários sólidos ou fluidos, a convecção ocorre entre uma superfície em contato com um fluido em movimento e a radiação ocorre entre duas superfícies a diferentes temperaturas.

Em uma tubulação de água quente sem isolamento térmico, onde a temperatura ambiente é inferior a temperatura da água, as transferências de calor ocorrem por convecção entre a água e a superfície interna do tubo, por condução através da parede do tubo, por convecção entre a superfície externa do tubo e o ar e por radiação entre a superfície externa da tubulação e uma superfície vizinha podendo ser, por exemplo, uma parede.

Para os cálculos da primeira parte deste trabalho, considerou-se apenas o processo de transferência de calor por condução na parede da tubulação para obtenção de resultados preliminares da pesquisa. 
A condução é a transferência de energia das partículas mais energéticas para as menos energéticas de uma substância devido às interações entre partículas. Esse modo de transferência de calor tem como mecanismo a difusão de energia devido ao movimento molecular aleatório (INCROPERA et al., 2008).

A taxa de transferência de calor por condução para uma tubulação sem isolamento térmico é expressa por meio da Equação 1:

$q_{r}=-\frac{2 k \pi L\left(T_{a}-T_{i}\right)}{\ln \left(R_{2} / R_{1}\right)}$

sendo:

$q_{r}$, a taxa de transferência de calor por condução na direção radial (W);

$k$, a condutividade térmica do material da tubulação $(\mathrm{W} / \mathrm{m} / \mathrm{K})$;

$L$, o comprimento do trecho de tubulação $(\mathrm{m})$;

$T_{i}$, a temperatura da água na tubulação $\left({ }^{\circ} \mathrm{C}\right)$;

$T_{a}$, a temperatura ambiente $\left({ }^{\circ} \mathrm{C}\right)$;

$R_{1}$, o raio interno da tubulação $(\mathrm{mm})$;

$R_{2}$, o raio externo da tubulação $(\mathrm{mm})$.

Para um trecho de tubulação de comprimento $L$, a temperatura da água no fim do trecho é expressa por meio da Equação 2:

$T_{i+1}=T_{i}-\frac{T_{i}-T_{a}}{Q \cdot c}\left[\frac{2 k \pi L}{\ln \left(R_{2} / R_{1}\right)}\right]$

sendo:

$T_{i+1}$, a temperatura da água no fim do trecho $\left({ }^{\circ} \mathrm{C}\right)$;

$T_{i}$, a temperatura da água no início do trecho $\left({ }^{\circ} \mathrm{C}\right)$;

$Q$, a vazão $(\mathrm{L} / \mathrm{s})$;

$c$, o calor específico da água $(\mathrm{J} / \mathrm{kg} / \mathrm{K})$.

A Equação 2 apresenta um processo iterativo, sendo possível determinar a variação da temperatura em " $n$ " distâncias ao longo da tubulação.

Figura 1 - Transferência de calor por condução através da tubulação

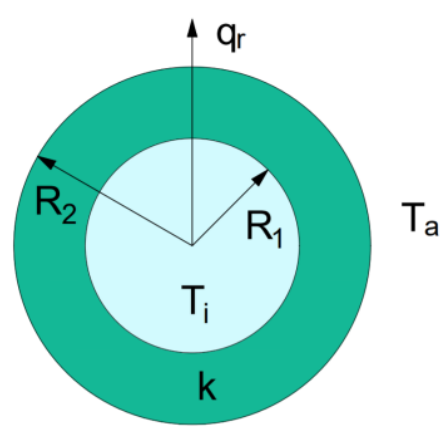

Fonte: Autor (2021)

\section{MÉTODO}

Para determinar o decaimento de temperatura na tubulação, foram feitas simulações numéricas utilizando o software Microsoft Excel ${ }^{(R)}$ e a licença estudantil do software Ansys Fluent $^{(R)}$, com intuito de comparar os resultados. 
O Ansys Fluent é um software de simulação que usa técnicas de Dinâmica dos Fluidos Computacionais (CFD do inglês, Computational Fluid Dynamics) e utiliza o método dos volumes finitos para obter as soluções das equações matemáticas que descrevem problemas que envolvem escoamentos de fluidos (VERSTEEG e MALALASEKERA, 2007).

A simulação no Excel consistiu em aplicar a Equação de Fourier de forma iterativa (Eq. 2) em trechos de 0,1 m de uma tubulação de 1,0 m de comprimento para cada diâmetro, considerando apenas o processo de transferência de calor por condução na parede do tubo.

Para a simulação no Ansys Fluent foi modelado uma tubulação de 1,0 m de comprimento para cada diâmetro transportando água quente e determinou-se a temperatura de saída da água no final do trecho estudado, considerando também a transferência de calor por convecção e radiação.

\section{RESULTADOS}

Os tubos de PPR PN 25 abordados neste trabalho possuem as dimensões especificadas pela NBR 15813-1 (ASSOCIAÇÃO BRASILEIRA DE NORMAS TÉCNICAS, 2018), apresentadas no Quadro 1.

Quadro 1 - Dimensões do tubo PPR PN 25

\begin{tabular}{|c|c|c|}
\hline Diâmetro nominal & Diâmetro externo $(\mathbf{m m})$ & Diâmetro interno $(\mathbf{m m})$ \\
\hline DN20 & 20 & 13,2 \\
\hline DN25 & 25 & 16,6 \\
\hline DN32 & 32 & 21,2 \\
\hline DN40 & 40 & 26,6 \\
\hline DN50 & 50 & 33,4 \\
\hline DN63 & 63 & 42,0 \\
\hline DN75 & 75 & 50,0 \\
\hline DN90 & 90 & 60,0 \\
\hline DN110 & 110 & 73,4 \\
\hline DN125 & 125 & 83,4 \\
\hline DN140 & 140 & 93,4 \\
\hline DN160 & 160 & 106,8 \\
\hline
\end{tabular}

Fonte: NBR 15813-1 (ASSOCIAÇÃO BRASILEIRA DE NORMAS TÉCNICAS, 2018)

Segundo Amanco (2010) e Tigre (2012), a $20^{\circ} \mathrm{C}$, a condutividade térmica do PPR é igual a $0,24 \mathrm{~W} / \mathrm{m} / \mathrm{K}$ e o calor específico é igual a $2000 \mathrm{~J} / \mathrm{kg} / \mathrm{K}$.

Para a determinação do decaimento de temperatura na tubulação no Excel, considerou-se os seguintes dados:

- tubulação linear sem isolamento térmico;

- comprimento da tubulação igual a 1,0 m; 
- vazão constante de $0,355 \mathrm{~L} / \mathrm{s}$ para todos os diâmetros, considerando-se como parâmetro $\mathrm{v}=2,5 \mathrm{~m} / \mathrm{s}$ para DN20;

- temperatura da água no início do trecho igual a $70^{\circ} \mathrm{C}$;

- temperatura ambiente constante e igual a $20^{\circ} \mathrm{C}$;

- propriedades termofísicas do PPR constante;

- calor específico da água constante e igual a $4191 \mathrm{~J} / \mathrm{kg} / \mathrm{K}$ (INCROPERA et al., 2008).

A Figura 2 apresenta os resultados obtido com a Equação 2 no Excel.

Figura 2 - Decaimento de temperatura ao longo do trecho

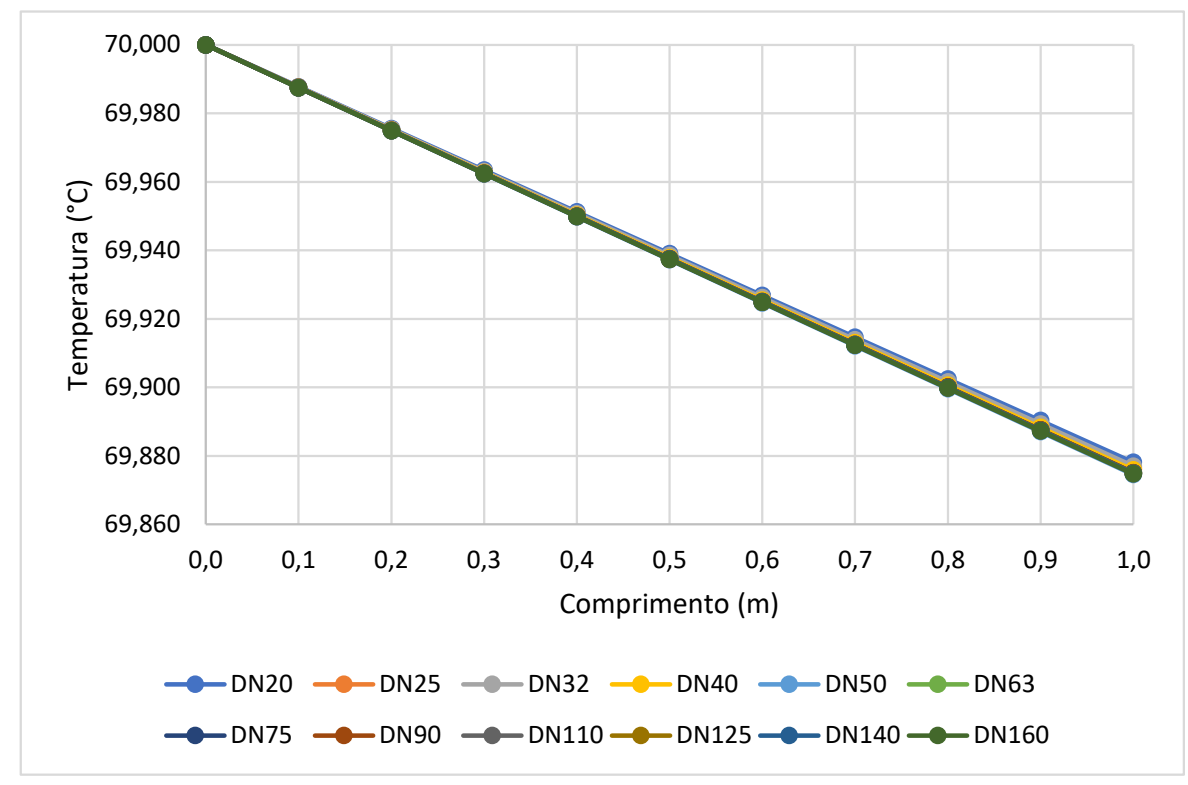

Fonte: Autor (2021)

Os resultados para temperatura no final do trecho obtido foram semelhantes, variando entre $69,875{ }^{\circ} \mathrm{C}$ para os tubos maiores e $69,878{ }^{\circ} \mathrm{C}$ para os menores. Essa semelhança pode ser explicada pelo fato de que os tubos PN 25 são da série $S 2,5$, que é um número adimensional que relaciona o diâmetro e a espessura de parede. Como esse valor calculado se assemelha para todos os tubos, a Equação 2, que depende da relação entre $R_{1}$ e $R_{2}$, apresenta resultados muito próximos para todos os diâmetros analisados.

Para a simulação do decaimento de temperatura no Ansys Fluent, considerou-se os mesmos dados usados no Excel. Para cada diâmetro de tubo foi feita uma simulação numérica com 1000 iterações. Usou-se o modelo de viscosidade laminar, porém foram feitas simulações utilizando também os modelos turbulentos $k-\varepsilon, k-\omega$ e SST com as configurações padrões do programa para o tubo DN20. Segundo Martins (2018), quanto maior a turbulência mais efetiva é a transferência de calor por convecção. Os modelos de turbulência usados modelam a energia cinética turbulenta $k$, sua dissipação $\varepsilon$, e a taxa de dissipação específica $\omega$, e são amplamente usados em problemas de engenharia. $O$ modelo $k-\omega$ entrega um tratamento preciso próximo à parede da tubulação, enquanto o modelo $k-\varepsilon$ possui um tratamento melhor nas regiões afastadas dela. Já modelo SST apresenta uma transição entre estes dois modelos (VERSTEEG e MALALASEKERA, 2007). 
A Figura 3 apresenta o gráfico do resultado da temperatura (curva vermelha) no fim do trecho da tubulação ao longo das iterações para o tubo DN20 em regime laminar.

Figura 3 - Temperatura da água no fim do trecho para o tubo DN20 (regime laminar)

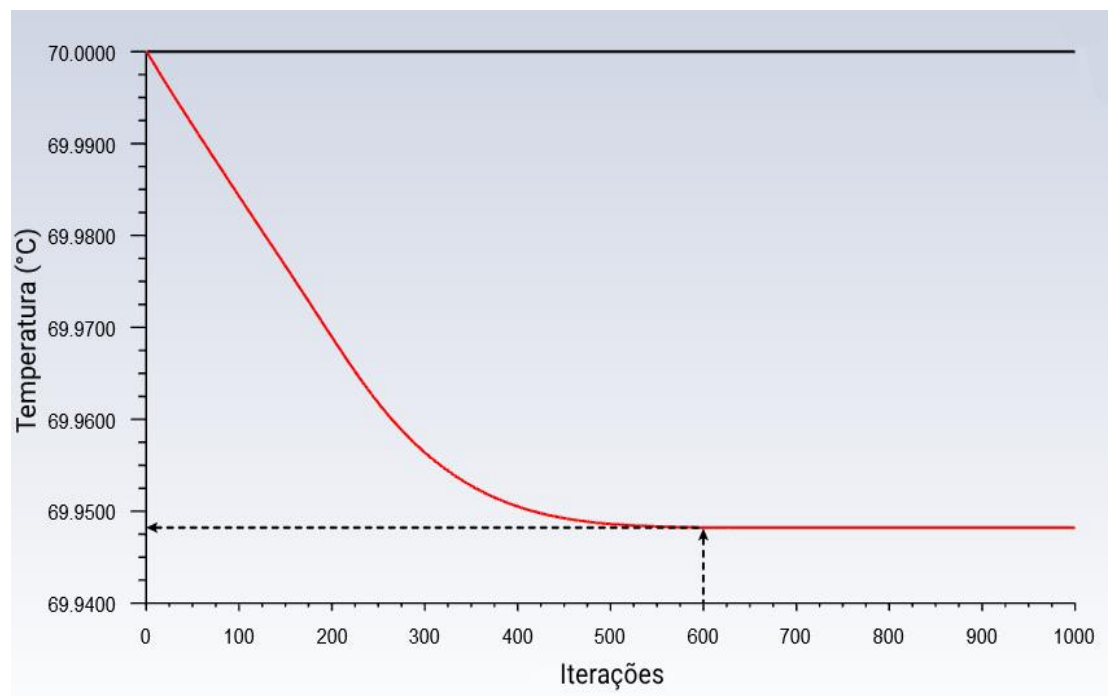

Fonte: Autor (2021)

Pelo gráfico, nota-se que foram necessárias aproximadamente 600 iterações para o resultado da temperatura convergir para o valor aproximado de $69,948{ }^{\circ} \mathrm{C}$. O número de iterações necessárias para os demais tubos foram diminuindo com o aumento do diâmetro, chegando a 200 iterações para o tubo DN160.

Na Tabela 1 são apresentados os resultados da temperatura no fim do trecho de 1,0 m obtidos no Excel e no Ansys Fluent para cada diâmetro, bem como as diferenças percentuais entre os valores.

Tabela 1 - Temperaturas no fim do trecho

\begin{tabular}{c|c|c|c}
\hline Diâmetro nominal & $\begin{array}{c}\text { Resultado obtido no } \\
\text { Excel }\left({ }^{\circ} \mathrm{C}\right)\end{array}$ & $\begin{array}{c}\text { Resultado obtido no } \\
\text { Ansys Fluent }\left({ }^{\circ} \mathrm{C}\right)\end{array}$ & $\begin{array}{c}\text { Diferença percentual } \\
\text { entre os resultados } \\
(\%)\end{array}$ \\
\hline DN20 & 69,878 & 69,948 & 0,100 \\
\hline DN25 & 69,876 & 69,939 & 0,090 \\
\hline DN32 & 69,877 & 69,925 & 0,069 \\
\hline DN40 & 69,876 & 69,911 & 0,050 \\
\hline DN50 & 69,875 & 69,896 & 0,030 \\
\hline DN63 & 69,875 & 69,878 & 0,004 \\
\hline
\end{tabular}


Tabela 1 - Temperaturas no fim do trecho (continuação)

\begin{tabular}{c|c|c|c}
\hline Diâmetro nominal & $\begin{array}{c}\text { Resultado obtido no } \\
\text { Excel }\left({ }^{\circ} \mathrm{C}\right)\end{array}$ & $\begin{array}{c}\text { Resultado obtido no } \\
\text { Ansys Fluent }\left({ }^{\circ} \mathrm{C}\right)\end{array}$ & $\begin{array}{c}\text { Diferença percentual } \\
\text { entre os resultados } \\
(\%)\end{array}$ \\
\hline DN75 & 69,875 & 69,866 & 0,013 \\
\hline DN90 & 69,875 & 69,848 & 0,039 \\
\hline DN110 & 69,875 & 69,832 & 0,062 \\
\hline DN125 & 69,875 & 69,819 & 0,080 \\
\hline DN140 & 69,875 & 69,810 & 0,093 \\
\hline DN160 & 69,875 & 69,798 & 0,110 \\
\hline
\end{tabular}

Fonte: Autor (2021)

Após os cálculos realizados pelo Ansys Fluent, foi plotado um corte longitudinal do tubo para visualização de um plano de temperaturas, apresentado na Figura 4. A região próxima a jusante possui cor quase idêntica à da região próxima a montante devido à escala de cores, que compreende valores entre $49,88^{\circ} \mathrm{C}$ e $70,00{ }^{\circ} \mathrm{C}$, e a temperatura a jusante, para o tubo da figura, é de $69,832{ }^{\circ} \mathrm{C}$, valor muito próximo de $70,00{ }^{\circ} \mathrm{C}$.

\section{Figura 4 - Plano de temperaturas do tubo DN110}

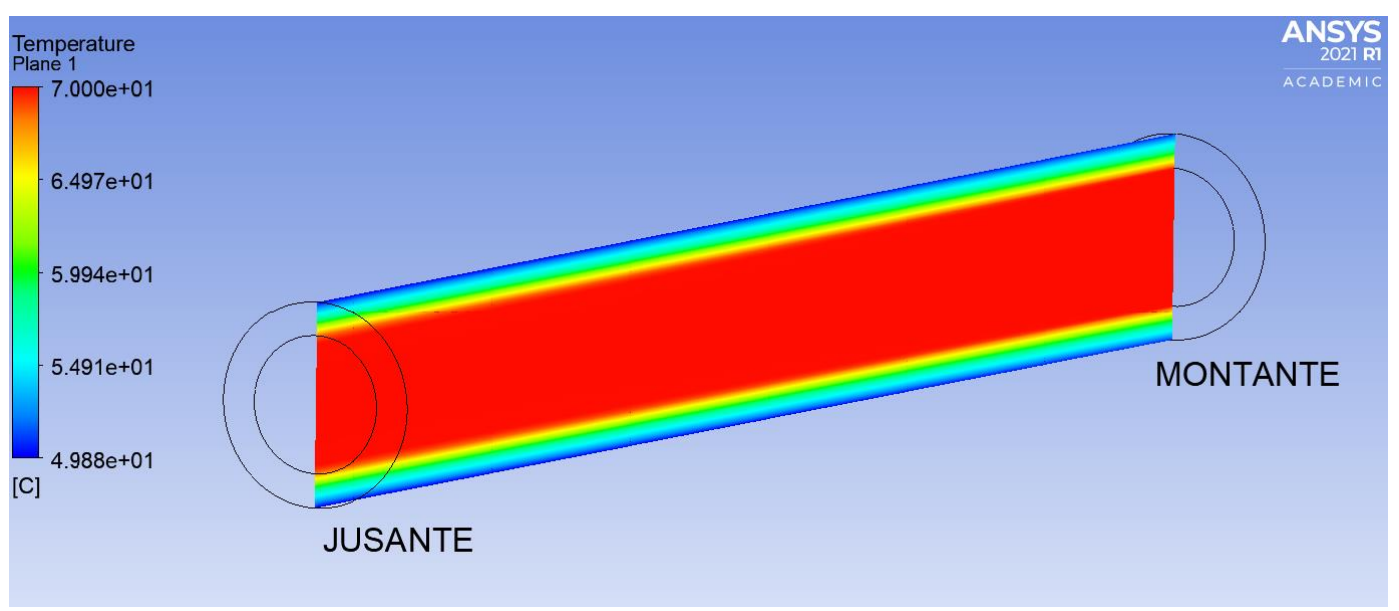

Fonte: Autor (2021)

As Figuras 5, 6 e 7 apresentam os gráficos dos resultados da temperatura (curva vermelha) no fim do trecho da tubulação ao longo das iterações para o tubo DN20 nos 3 modelos de turbulência utilizados. 
Figura 5 - Temperatura da água no fim do trecho para o tubo DN20 (modelo $k-\varepsilon$ )

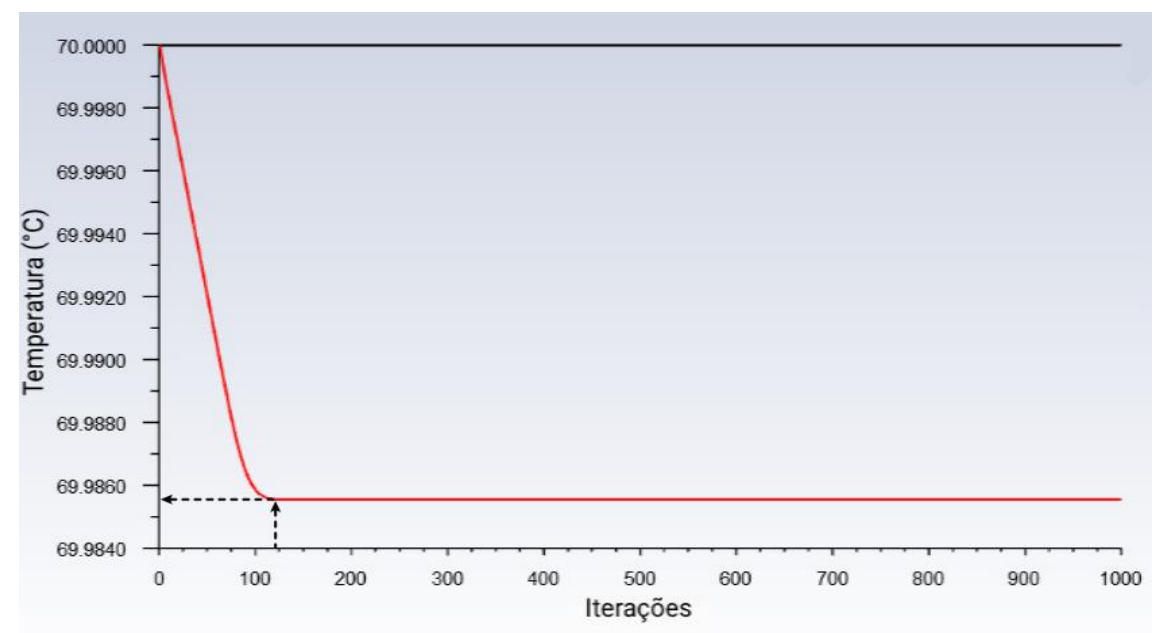

Fonte: Autor (2021)

Figura 6 - Temperatura da água no fim do trecho para o tubo DN20 (modelo $k-\omega)$

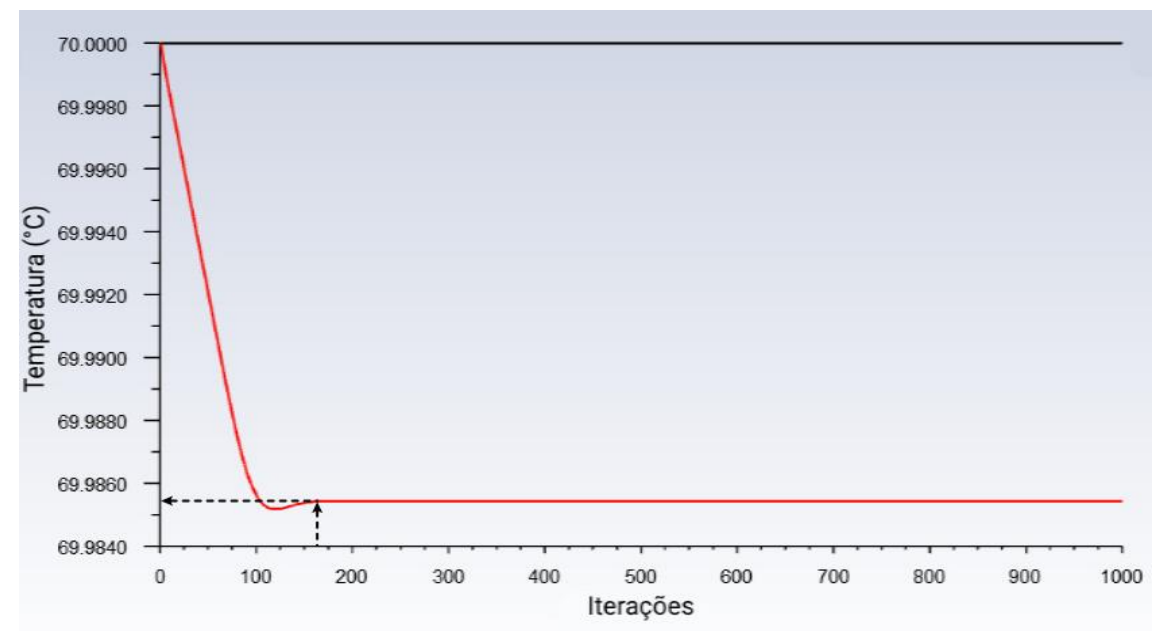

Fonte: Autor (2021)

Figura 7 - Temperatura da água no fim do trecho para o tubo DN20 (modelo SST)

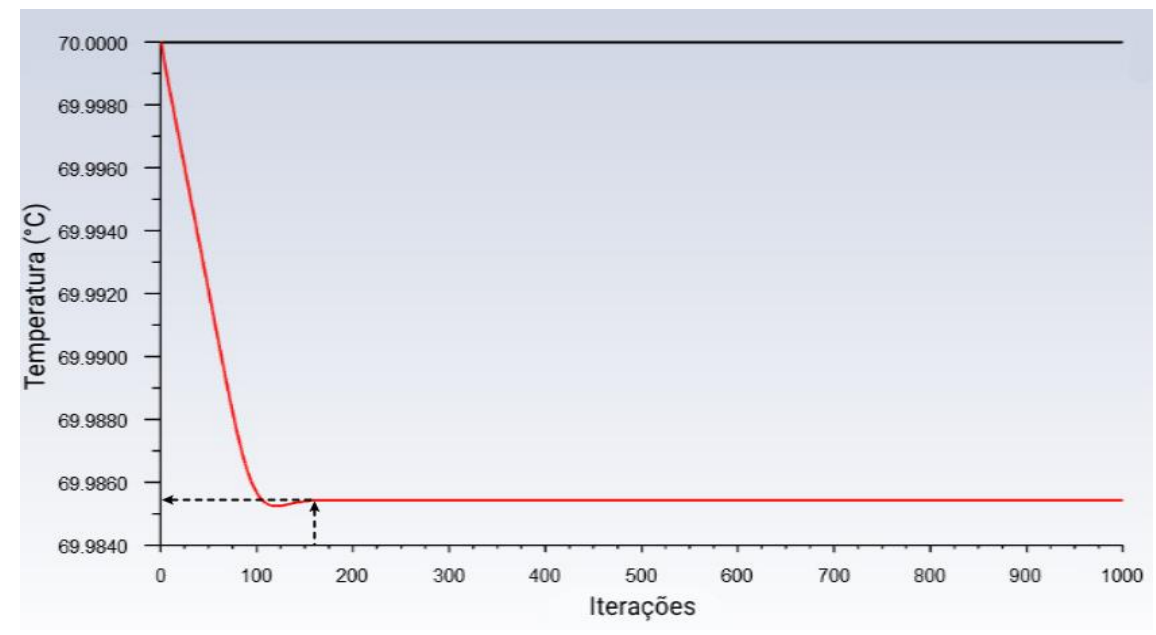

Fonte: Autor (2021) 
$\mathrm{Na}$ Tabela 2 são apresentados os resultados da temperatura no fim do trecho de 1,0 m obtidos no Ansys Fluent para o tubo DN20, bem como as diferenças com os resultados do Excel.

Tabela 2 - Temperaturas no fim do trecho

\begin{tabular}{c|c|c}
\hline Modelo de viscosidade & $\begin{array}{c}\text { Resultado obtido no Ansys } \\
\text { Fluent }\left({ }^{\circ} \mathbf{C}\right)\end{array}$ & $\begin{array}{c}\text { Diferença percentual entre os } \\
\text { resultados do Ansys Fluent e } \\
\text { Excel (\%) }\end{array}$ \\
\hline Laminar & 69,948 & 0,100 \\
\hline$k-\varepsilon$ & 69,986 & 0,154 \\
\hline$k-\omega$ & 69,985 & 0,153 \\
\hline SST & 69,985 & 0,153 \\
\hline
\end{tabular}

Fonte: Autor (2021)

\section{CONCLUSÃO}

Devido a importância do correto dimensionamento de sistemas de água quente, o presente trabalho visa contribuir com dados sobre o decaimento de temperatura do tubo PPR PN 25 em função do diâmetro, os quais não são especificados nos catálogos dos fabricantes.

Os cálculos realizados no Excel contemplaram apenas o modo de transferência de calor por condução, enquanto para a análise no Ansys Fluent considerou também a convecção e a radiação. Os resultados obtidos nos dois programas apresentaram diferenças menores que $0,2 \%$, sendo que as maiores diferenças $(0,154 \%)$ ocorreram na análise com regime turbulento no Ansys Fluent. Dessa forma, é necessário um estudo no Excel considerando convecção e radiação e a comparação com os modelos sugeridos no Ansys para a maior precisão da simulação.

\section{REFERÊNCIAS}

AMANCO. Manual Técnico: Linha Amanco PPR. Joinville, set. 2010. Disponível em: http://assets.production.amanco.com.br.s3.amazonaws.com/uploads/gallery_asset/file/37/baixa_a mco_atualizacao_manual_tecnico_amanco_PPR_2010_v11.pdf. Acesso em: 25 maio 2021.

ASSOCIAÇÃO BRASILEIRA DE NORMAS TÉCNICAS. NBR 15813-1: Sistemas de tubulações plásticas para instalações prediais de água quente e fria Parte 1: Tubos de polipropileno copolímero random PP-R e PP-RCT — Requisitos. Rio de Janeiro: ABNT, 2018.

BORGNAKKE, C.; SONNTAG, R. E. Fundamentos da termodinâmica. Tradução da $8^{a}$ edição norteamericana. São Paulo: Blucher, 2018. E-book (730 p.). ISBN 978-85-212-0793-1.

DANIELETTO, J. R. B. Manual de tubulações de polietileno e polipropileno: características, dimensionamento e instalação. 1. ed. São Paulo: 2007. 528 p.

INCROPERA, F. P. et al. Fundamentos de transferência de calor e de massa. 6. ed. Rio de Janeiro: LTC, 2008. 643 p. ISBN 978-85-216-1584-2.

MARTINS, J. R. Análise numérica da dissipação de calor do motor elétrico do sistema BCSS em skid. 2018. 144 f. Dissertação (Mestrado em Energia) - Programa de Pós-Graduação em Energia, 
Universidade Federal do Espírito Santo, Centro Universitário Norte do Espírito Santo, São Mateus, 2018.

TIGRE. PPR: Termofusão: Catálogo Técnico. Joinville, jul. 2012. Disponível em: https://www.tigre.com.br/themes/tigre2016/downloads/catalogos-tecnicos/ct-ppr-termofusao.pdf. Acesso em: 31 maio 2021.

VERSTEEG, H. K.; MALALASEKERA, W. An Introduction to Computational Fluid Dynamics: The Finite Volume Method. 2. ed. Inglaterra: Pearson Education, 2007. 503 p. ISBN 978-0-13-127498-3. 